\title{
Consistent Structural Linearization in Flexible Aircraft Dynamics with Large Rigid-Body Motion
}

\author{
Henrik Hesse* Joseba Murua $^{\dagger}$ and Rafael Palacios ${ }^{\ddagger}$ \\ Imperial College, London SW7 2AZ, United Kingdom
}

\begin{abstract}
This paper investigates the linearization, using perturbation methods, of the structural deformations in the nonlinear flight dynamic response of aircraft with slender, flexible wings. This has been achieved by first coupling a displacement-based, geometricallynonlinear flexible-body dynamics formulation with the 3D Unsteady Vortex Lattice Method, followed by a consistent linearization of the structural degrees of freedom, which are assumed to be small in a body-fixed reference frame. The translations and rotations of that reference frame can be arbitrarily large, however. The resulting system preserves all couplings between rigid and elastic motions and can be projected onto a few vibration modes of the unconstrained aircraft with arbitrarily-large, geometrically-nonlinear deformation at a trim condition. The dynamics of the system are then written in tensor form, with up to cubic terms due to the nonlinear rigid-body terms, and with a limited number of coefficients that can be pre-computed prior to the time-marching simulation. Numerical studies on a representative HALE UAV are presented to illustrate the approach and results are compared to the mean-axes solution.
\end{abstract}

\section{Nomenclature}

$\begin{array}{ll}\text { Symbols } & \\ A & \text { aerodynamic influence coefficient matrix } \\ C^{B A} & \text { coordinate transformation matrix from frame } A \text { to } B \\ \mathcal{C} & \text { global tangent damping matrix } \\ F & \text { nodal external forces } \\ I & \text { unit matrix } \\ K & \text { sectional local curvature } \\ \mathcal{K} & \text { global tangent stiffness matrix } \\ M & \text { nodal external moments } \\ \mathcal{M} & \text { global tangent mass matrix } \\ N & \text { matrix of shape functions } \\ p & \text { generalized displacements in modal basis } \\ Q & \text { global vector of generalized forces } \\ r & \text { position vector of the origin of body-fixed frame, } A \\ R & \text { local position vector along the beam reference line } \\ s & \text { arc-length along beam reference line } \\ T & \text { tangential operator } \\ u & \text { input vector } \\ v & \text { inertial translational velocity of the body-fixed frame, } A\end{array}$

*Graduate Student, Department of Aeronautics, AIAA Student Member.

$\dagger$ Graduate Student, Department of Aeronautics, AIAA Student Member.

${ }^{\ddagger}$ Lecturer, Department of Aeronautics (Contact author: rpalacio@imperial.ac.uk), AIAA Member. 


\begin{tabular}{|c|c|}
\hline$V$ & inertial translational velocity at a beam cross section \\
\hline$x$ & state vector \\
\hline$y$ & output vector \\
\hline$\beta$ & vector of global translational and rotational velocities \\
\hline$\gamma$ & beam local force strain \\
\hline$\Gamma$ & circulation strength of a vortex ring \\
\hline$\zeta$ & quaternion for global orientation of the body-fixed frame, $A$ \\
\hline$\eta$ & vector of nodal displacements and rotations \\
\hline$\Theta$ & Euler angles \\
\hline$\kappa$ & beam local moment strain \\
\hline$\nu$ & global displacements and rotations as time integral of $\beta$ \\
\hline$\xi$ & beam cross-sectional coordinates \\
\hline$\Phi$ & matrix of mode shapes \\
\hline$\delta \phi$ & global virtual rotations \\
\hline$\delta \Phi$ & local virtual rotations \\
\hline$\chi$ & coordinates of the aerodynamic lattice \\
\hline$\Psi$ & local Cartesian Rotation Vector (CRV) along the beam reference line \\
\hline$\omega$ & inertial angular velocity of the body-fixed frame, $A$ \\
\hline$\Omega$ & inertial angular velocity at a beam cross section \\
\hline \multicolumn{2}{|c|}{ Subscripts } \\
\hline$a e$ & aerodynamic \\
\hline$A$ & body-fixed reference frame \\
\hline$b$ & bound, corresponding to lifting surface \\
\hline$B$ & local reference frame on flexible members \\
\hline$G$ & inertial reference frame \\
\hline$R$ & rigid-body \\
\hline$S$ & structural \\
\hline$w$ & wake \\
\hline \multicolumn{2}{|c|}{ Superscripts } \\
\hline$\dot{\bullet}$ & derivatives with respect to time, $t$ \\
\hline$\bullet$ & derivatives with respect to arc-length, $s$ \\
\hline • & skew-symmetric operator \\
\hline $\bar{\bullet}$ & small perturbations around an equilibrium \\
\hline
\end{tabular}

\section{Introduction}

Design trends for next-generation passenger aircraft feature more slender and longer wings to increase aerodynamic efficiency and achieve further weight reduction. Two striking examples to demonstrate this development are the Boeing SUGAR Volt and MIT "Double Bubble" D8 concepts. Both designs include wings of very high aspect ratio, which results in lighter, more flexible platforms with potential couplings between rigid-body and structural dynamics characteristics. Hence, a better understanding of the coupled aeroelastic and flight dynamics response of such platforms can improve the design process and is crucial for the design of control systems to optimize their performance. ${ }^{1}$

The most common approach in flight dynamics to introduce structural flexibility into the vehicle dynamics is the mean-axes assumption. ${ }^{2}$ It assumes that there exists an instantaneous reference frame which decouples the structural and rigid-body dynamics degrees of freedom (DoF), thus providing a much simplified description that superimposes elastic deformations to the rigid-body dynamics. This approach has been followed in a number of studies, ${ }^{3-5}$ mostly for relatively stiff vehicles. By hypothesizing the existence of mean 
axes, the Newton-Euler equations can be used to replace the rigid-body modes of the rigid structures, leading to the aforementioned set of decoupled equations of motion (EoM). The 3-D unsteady aerodynamic loads are obtained as the corresponding generalized forces for the structural mode shapes, and can be computed through a variety of methods. The most common aerodynamic model is probably the doublet-lattice method (DLM) ${ }^{6}$ which provides aerodynamic influence coefficients at a set of reduced frequencies assuming harmonic displacements on the natural vibration modes of the structure. These frequency-domain coefficients are then curve-fitted into state-space time-domain models through rational function approximations. ${ }^{7}$

Higher fidelity aerodynamic models have also been used, but field methods are still very demanding for unsteady flows with large motions of the solid boundaries, as they require the creation and distortion of the mesh as the structure deforms. Hence, they have been scarcely employed in flexible aircraft dynamics. ${ }^{8-10}$ In fact, many authors have resorted to 2D strip theory aerodynamics for coupled aircraft-dynamics studies. ${ }^{11-18}$ While strip theory offers a computationally efficient approach that has led to the identification of crucial features in flexible vehicles, it neglects relevant 3-D flow physics. In this context, the 3D Unsteady Vortex-Lattice Method (UVLM) ${ }^{19}$ constitutes a medium-fidelity approach $^{20}$ that brings excellent compromise for flexible aerial platforms: it enforces the boundary conditions on the deformed shape of the lifting surfaces, thus capturing geometrically-nonlinear deformations and models the force-free wake and aerodynamic interference. ${ }^{21}$ In turn, the linearization of the equations leads to a compact and efficient state-space formulation, ideally suited for multidisciplinary integration, stability analysis and control synthesis. The linearized version of the UVLM could substitute the DLM in virtually any aeroelastic simulation, as it offers the same degree of fidelity but caters for in-plane motions, non-planar prescribed wakes, and large static deflections. Since it is based on potential-flow theory, as the DLM and strip theory, the method is however not suitable to predict viscous drag, when the wing enters stalled conditions, or at low Reynolds number.

Even at low velocities the dynamic load levels due to atmospheric turbulence particularly at lower altitudes require a complex aeroelastic balancing to minimize the instantaneous wing loading in highly efficient designs and can create large, geometrically-nonlinear wing deformations. Hence, more recent work $^{11,15,17,21-23}$ has been concerned with coupling different aerodynamic models, mostly strip theory or the UVLM, with geometrically-nonlinear structural models to include the effects of elastic deformations on the flight dynamics of flexible aircraft. Typically, the displacements and rotations of each point along the structure are the primary variables in the numerical solution, ${ }^{24-26}$ where the elastic DoF are defined in terms a global body-fixed reference frame attached to the reference configuration to include the rigid-body motion of the vehicle. Alternative solutions to the displacement-based approach exist using the strain of the beam elements as primary variables, ${ }^{15,17,27}$ or taking both the local velocities and strains (the intrinsic description) as independent degrees of freedom ${ }^{28-30}$ - the latter can provide some numerical advantages on aircraft-type geometries. A comparative study on these different structural models for flexible-aircraft dynamics was presented in Palacios et al. ${ }^{20}$

These coupled geometrically-nonlinear frameworks allow capturing the large effect that structural deformations may have on the aircraft dynamics characteristics ${ }^{15}$ and stability properties. ${ }^{31}$ However, the resulting system of equations is highly nonlinear and numerically stiff because of large rigid-body variables and comparably small elastic deformations. This raises the question of the required fidelity of the aeroelastic model tailored to the physics of the problem at hand in order to capture the effect of elastic deformations efficiently. Hence, this work aims to provide efficient simulation tools for flexible aircraft dynamics that still accurately captures the dominant dynamic features.

Previous work by the authors ${ }^{32}$ focused on the consistent structural linearization of the structural DoF in nonlinear flexible-body dynamics problems with small elastic deformations under prescribed forces. The resulting formulation allowed projection of the nonlinear EoM onto the vibration modes of the unconstrained structure to reduce the problem size while keeping the nonlinearity in the rigid-body dynamics equations and the coupling between rigid-body and structural dynamics. This approach resulted in closed-form expressions for all the modal coefficients in the equations that only need to be computed once and give an insight into the coupling effects between elastic and structural DoF. The resulting formulation was used to investigate the coupling effect of the nonlinear gyroscopic forces on the elastic deformations to assess the mean-axes approximation on an unconstrained flexible multi-beam problem without aerodynamics. Whereas the rigidbody response always converged to the rigid case for very stiff problems, the mean-axes approach failed to predict the elastic deformations accurately for the analyzed problem of rotational nature. It was shown that these results are independent of the level of deformation.

This work presents the integration of linear-flexible/nonlinear-rigid formulation into the framework for 
Simulation of High-Aspect Ratio Planes (SHARP) ${ }^{21,31-33}$ to analyze the impact of independent linearization of structural DoF and aerodynamics on the prediction of flight dynamics of flexible aircraft. In particular, the linearized form of the discrete-time state-space UVLM ${ }^{31,34}$ is integrated with the modal form of the flexiblebody dynamics equations. This reduces the size of the unconstrained aeroelastic system dramatically, but keeps the nonlinearities in the 6-DoF aircraft rigid-body dynamics. Numerical examples present this novel approach on the open-loop response of a HALE aircraft perturbed from its trim condition, which is compared to the linear stability characteristics of the aircraft using the stability tool in SHARP. Comparison to the mean-axes solution will finally give an insight into the effect of neglecting some effects of the gyroscopic forces on the elastic deformations and aircraft flight dynamics.

\section{Flexible-Body Dynamics}

The flexible vehicle will be modeled using geometrically-nonlinear composite beam elements on a moving (body-attached) frame of reference. The elastic degrees of freedom are the displacements and the Cartesian Rotation Vector (CRV) at the element nodes, with respect to the body-frame, and the rigid-body motions are described by the translational and angular velocities of that frame. The nonlinear equations of motion are linearized with respect to the elastic degrees of freedom using perturbation methods. They can then be projected onto a set of elastic modes of the unrestrained system at some reference condition, which will give a compact form of the equations of motion, but that still includes all nonlinear effects due to the large rigid-body velocities. The details of this approach can be found in Hesse and Palacios ${ }^{32}$ and this section will only present the main results.

\section{II.A. Geometrically-Exact Displacement-Based Formulation}

As shown in Fig. 1, the deformation of the structure is described in terms of a moving, body-fixed reference coordinate system, $A$, which moves with respect to an inertial (ground) frame, $G$, by the inertial translational and angular velocities, $v_{A}(t)$ and $\omega_{A}(t)$, of its origin ${ }^{\mathrm{a}}$. The orientation of the global frame, $A$, with respect to the inertial frame, $G$, is given by the coordinate transformation matrix $C^{G A}(t)$. The local orientation along the beam reference line is defined by a local coordinate system, $B$, and is parametrized by the CRV, $\Psi(s, t) .^{35}$ The corresponding coordinate transformation matrix between the global body-fixed reference frame, $A$, and the local deformed frame, $B$, is $C^{B A}(s, t)=C(\Psi)$.

The nodal positions, $R_{A}(s, t)$, and the cross-sectional orientations, $\Psi(s, t)$, thus form the independent set of variables. The deformation of the reference line going from the undeformed state at $t=0$ to the current state at time $t$ is given by the force and moment strains, ${ }^{36}$ which are written as

$$
\begin{aligned}
& \gamma(s, t)=C(\Psi(s, t)) R_{A}^{\prime}(s, t)-C(\Psi(s, 0)) R_{A}^{\prime}(s, 0), \\
& \kappa(s, t)=T(\Psi(s, t)) \Psi^{\prime}(s, t)-T(\Psi(s, 0)) \Psi^{\prime}(s, 0) .
\end{aligned}
$$

where $(\bullet)^{\prime}$ is the derivative with respect to the arc-length $s$, and $T(\Psi)$ is the tangential operator. ${ }^{35}$ Analogously, the local translational and angular inertial velocities are given, respectively, as

$$
\begin{aligned}
V_{B}(s, t) & =C(\Psi(s, t)) \dot{R}_{A}(s, t)+C(\Psi(s, t))\left[v_{A}(t)+\widetilde{w}_{A}(t) R_{A}(s, t)\right], \\
\Omega_{B}(s, t) & =T(\Psi(s, t)) \dot{\Psi}(s, t)+C(\Psi(s, t)) \omega_{A}(t),
\end{aligned}
$$

where $(\boldsymbol{\bullet})$ is the derivative with respect to time and $(\tilde{\bullet})$ the cross-product (or skew-symmetric) operator.

This geometrically-exact beam kinematics description serves to express the kinetic and potential energy of the system in terms of the beam displacement and rotations. ${ }^{20,35}$ A finite-element discretization is then introduced on both variables (in particular, 2- and 3-noded elements have been implemented), which gives the discrete form of the nonlinear equations of motion. If $\eta$ is the column vector with all the nodal displacements and rotations and $\beta^{\top}=\left\{\begin{array}{ll}v_{A}^{\top} & \omega_{A}^{\top}\end{array}\right\}$, the dynamic equations are written as ${ }^{32}$

$$
\mathcal{M}(\eta)\left\{\begin{array}{c}
\ddot{\eta} \\
\dot{\beta}
\end{array}\right\}+\left\{\begin{array}{c}
Q_{g y r}^{S} \\
Q_{g y r}^{R}
\end{array}\right\}+\left\{\begin{array}{c}
Q_{s t i f}^{S} \\
0
\end{array}\right\}=\left\{\begin{array}{l}
Q_{e x t}^{S} \\
Q_{e x t}^{R}
\end{array}\right\},
$$

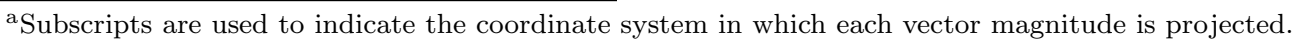




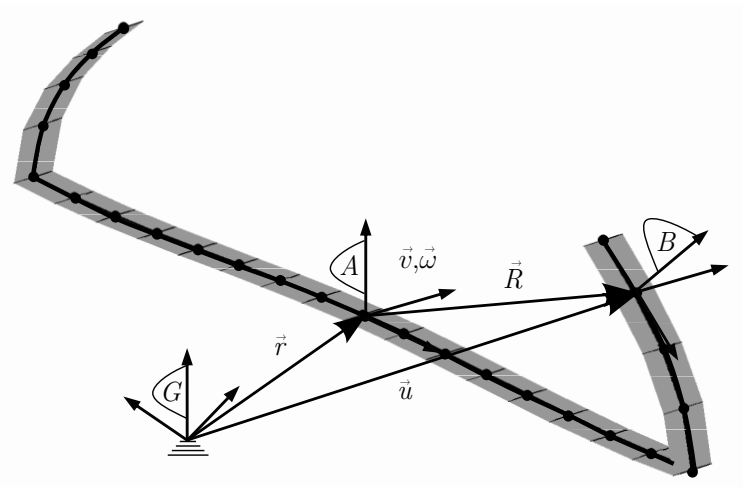

Figure 1: Multi-beam configuration with the definition of reference frames.

where structural and rigid-body components (denoted by superscripts $S$ and $R$ ) have been identified in the gyroscopic, stiffness and external forces. These differential equations couple the nonlinear beam dynamics and the nonlinear rigid-body dynamics through the mass matrix, $\mathcal{M}(\eta)$, and the discrete gyroscopic forces, $Q_{g y r}(\eta, \dot{\eta}, \beta)$. Additional coupling occurs with follower forces (e.g., the aerodynamic loads). Equation (3) is solved together with the propagation equations that determine the position and orientation of the body-fixed reference frame. ${ }^{37}$ The equations have been solved here using a implicit Newmark- $\beta$ integration scheme.

\section{II.B. Linearization of Elastic Degrees of Freedom in Nonlinear Flexible-Body Dynamics}

The particular case of small structural deformations about a large equilibrium condition is considered next. The (large) elastic deformations at the static equilibrium condition will be refer to as before as $\eta$, while $\bar{\eta}(t)$ will be the small elastic deformations that occur together with the (not-necessarily small) rigid-body velocities $\beta(t)$. With those assumptions, perturbation of the elastic degrees of freedom in Eq. (3) gives linear inertial and elastic terms, which only depend on the deformed shape, while the gyroscopic forces require particular attention because of the coupling between rigid-body and elastic states. This results in $^{32}$

$$
\mathcal{M}(\eta)\left\{\begin{array}{c}
\ddot{\bar{\eta}} \\
\dot{\beta}
\end{array}\right\}+\overline{\mathcal{C}}(\eta, \beta)\left\{\begin{array}{c}
\dot{\bar{\eta}} \\
\beta
\end{array}\right\}+\overline{\mathcal{K}}(\eta, \beta)\left\{\begin{array}{l}
\bar{\eta} \\
0
\end{array}\right\}=Q_{\text {ext }}(\bar{\eta}, \dot{\bar{\eta}}, \beta, \zeta)+\text { H.O.T. }
$$

where $\overline{\mathcal{C}}$ and $\overline{\mathcal{K}}$ are the updated damping and stiffness matrices, which originate from the perturbation of the equations on the elastic degrees of freedom. It is important to note that the mass matrix $\mathcal{M}$ only depends on the static deformations, $\eta$, but the damping and stiffness matrices are functions of $\eta$ and also of the instantaneous rigid-body velocity, $\beta$ (with linear and quadratic dependencies on that variable ${ }^{32}$ ).

In the next section, a reduction of the system size will be obtained by projecting the structural variables onto the natural vibration modes of a reference linear system. This requires linearization of the full dynamic system which will be done around a static equilibrium condition, $Q_{s t i f}(\eta)=Q_{e x t}(\eta, \zeta)$, with possibly large elastic deformations, $\eta$, at the reference condition. Again, small changes from this state will be represented with over-bars, that is, $(\bar{\eta}, \dot{\bar{\eta}}, \ddot{\bar{\eta}}, \bar{\beta}, \dot{\bar{\beta}}, \bar{\zeta})$ and the linearized (incremental) form of Eq. (4) is

$$
\mathcal{M}(\eta)\left\{\begin{array}{l}
\ddot{\bar{\eta}} \\
\ddot{\bar{\nu}}
\end{array}\right\}+\mathcal{K}(\eta)\left\{\begin{array}{l}
\bar{\eta} \\
\bar{\nu}
\end{array}\right\}=\bar{Q}_{\text {ext }}(\overline{\bar{\eta}}, \dot{\bar{\eta}}, \dot{\bar{\nu}}, \bar{\zeta}),
$$

where we have introduced the new variable $\bar{\nu}$ which integrates the rigid-body velocities such that $\dot{\bar{\nu}}=\bar{\beta}$. This has no effect on the solution of the linear equation since the rigid-body terms have no stiffness, but the change in variables enables the computation of vibration modes of the unconstrained structure. The constant tangent stiffness matrix, $\mathcal{K}$, is obtained through direct linearization of $\overline{\mathcal{K}}$ with respect to the rigidbody velocities around the equilibrium $\beta=0$. Note however that the equations are invariant with respect to translational velocities and this equations determine the dynamics for a trimmed vehicle in forward flight. This coupled linear equations of motion will be the basis for aeroelastic stability analyses. 


\section{II.C. Modal Projection of the Perturbed Equations}

Under the assumption of small elastic deformations, it is possible to write the nonlinear system EoM, Eq. (4), in terms of global shape functions by projecting the dynamics equations on the modes of the unconstrained structure. These vibration modes are obtained from the unforced fully-linearized EoM, Eq. (5), such that

$$
\left\{\begin{array}{l}
\bar{\eta} \\
\bar{\nu}
\end{array}\right\}=\Phi\left\{\begin{array}{c}
p \\
\bar{\nu}^{*}
\end{array}\right\}
$$

where $p$ is the vector of the projected modal coordinates and $\Phi$ is the matrix of the corresponding mode shapes, which include the six rigid-body modes and the remaining elastic mode shapes describe the motion of the body-fixed frame, $A$, and the elastic deformation with respect to this moving frame. Hence, the vector of modal rigid-body displacements and rotations, $\nu^{*}$, is a linear map of the motion of the body-fixed frame, $A$, and the displacement of this frame due to the elastic mode shapes. The structural and rigid-body velocities/accelerations can now be expressed in terms of the modal basis, such that

$$
\left\{\begin{array}{c}
\dot{\bar{\eta}} \\
\beta
\end{array}\right\}=\Phi\left\{\begin{array}{c}
\dot{p} \\
\beta^{*}
\end{array}\right\} \quad \text { and } \quad\left\{\begin{array}{c}
\ddot{\bar{\eta}} \\
\dot{\beta}
\end{array}\right\}=\Phi\left\{\begin{array}{c}
\ddot{p} \\
\dot{\beta}^{*}
\end{array}\right\},
$$

which is substituted in Eq. (4) to obtain the modal form of the coupled EoM for this system, as

$$
\Phi^{\top} \mathcal{M}(\eta) \Phi\left\{\begin{array}{c}
\ddot{p} \\
\dot{\beta}^{*}
\end{array}\right\}+\Phi^{\top} \overline{\mathcal{C}}(\eta, \beta) \Phi\left\{\begin{array}{c}
\dot{p} \\
\beta^{*}
\end{array}\right\}+\Phi^{\top} \overline{\mathcal{K}}(\eta, \beta) \Phi\left\{\begin{array}{c}
p \\
0
\end{array}\right\}=\Phi^{\top} Q_{e x t}(\bar{\eta}, \dot{\bar{\eta}}, \dot{\nu}, \zeta) .
$$

The modal damping and stiffness matrices remain functions of the arbitrarily-large rigid-body DoF and would need to be recomputed for every $\beta$. If we make use of the fact that the damping matrix $\overline{\mathcal{C}}$ and stiffness matrix $\overline{\mathcal{K}}$ have, respectively, linear and quadratic dependencies with the rigid-body DoF, then it is possible to write the modal matrices in terms of third and fourth-order tensors such that

$$
\begin{aligned}
\Phi_{i j} \overline{\mathcal{C}}_{j k} \Phi_{k l} & =c_{i l r} \beta_{r}(t), \\
\Phi_{i j} \overline{\mathcal{K}}_{j k} \Phi_{k l} & =k_{i l}^{\text {sif }}+k_{i l r s}^{\text {gyr }} \beta_{r}(t) \beta_{s}(t),
\end{aligned}
$$

where we sum over repeated indeces and have identified the contributions to modal stiffness matrix from elastic and gyroscopic forces. The tensors $c$ and $k$ are constant in time and their dimensions are $i, l=$ $\{1, \ldots, m\}$ and $r, s=\{1, \ldots, 6\}$ for $m$ number of modes used in the expansion. This approach reduces the size of the structural subsystem dramatically while keeping the nonlinearities in the rigid-body degrees of freedom.

\section{Coupled Aeroelasticity and Flight Dynamics Model}

The previous flexible-body dynamics description is the core of a computer framework for the Simulation of High-Aspect-Ratio Planes (SHARP). The 3-D unsteady aerodynamics are obtained using the Unsteady Vortex-Lattice Method (UVLM), which is able to solve wing surfaces undergoing large deformations. As part of the solution process the free wake is computed which also allows the evaluation of interference effects. ${ }^{21}$ Details on implementation and validation of the UVLM and mapping between the aerodynamic and structural grid in SHARP have been presented by Murua et al. ${ }^{31}$ and only a summary is presented here.

\section{III.A. Linearized Unsteady Vortex-Lattice Method}

The Unsteady-Vortex Lattice Method is a 3D potential-flow method. As in other panel methods, elementary (singularity) solutions are distributed over a surface and enforcement of the impermeability boundary condition on solid boundaries leads to a system of algebraic equations. In the UVLM, vortex-ring quadrilateral elements are used to discretize both lifting surfaces and wakes. These elements are antisymmetric singularities that ignore thickness and are placed on the mean surface. Each surface (bound) vortex-ring has a associated a circulation strength and a collocation point, at which the boundary condition is satisfied. The vortex rings are displaced a quarter-chord length from the actual geometry, and the collocation points lie at the center of the element. 
In its general form, the UVLM is a nonlinear method in which the shape of a force-free wake is obtained as part of the solution procedure, usually using a first-order explicit integration scheme. Aerodynamic influence coefficients that include the influence of all surfaces and wakes over all collocation points are computed through the Biot-Savart law. This leads to the system of equations for the unknown circulation strengths. Once the vorticity distribution is computed at each time step, the aerodynamic pressures can be computed using Bernoulli's equation. Note that, as the influence coefficients can account for large deflections of the aerodynamic surfaces, the method is also geometrically nonlinear. This numerical algorithm, based on an explicit time-stepping technique, is extensively described by Katz and Plotkin. ${ }^{19}$

The governing equations can also be linearized, leading to a compact set of discrete-time state-space equations. Based on the ideas outlined by Hall, ${ }^{38}$ the equations are linearized performing small perturbation expansions and assuming a frozen aerodynamic geometry ${ }^{31}$ leading naturally to a descriptor state-space form of the UVLM given by

$$
\begin{aligned}
E_{a e} \Delta x_{a e}^{n+1}+F_{a e} \Delta u_{a e}^{n+1} & =A_{a e} \Delta x_{a e}^{n}+B_{a e} \Delta u_{a e}^{n} \\
\Delta y_{a e}^{n} & =C_{a e} \Delta x_{a e}^{n}+D_{a e} \Delta u_{a e}^{n}
\end{aligned}
$$

where superscripts $n$ and $n+1$ refer to the current and next time steps. The outputs $y_{a e}$ are the aerodynamic loads, and the states, $x_{a e}$, and inputs, $u_{a e}$, of the linearized aerodynamic system are given by

$$
x_{a e}=\left\{\begin{array}{c}
\Gamma_{b} \\
\Gamma_{w} \\
\dot{\Gamma}_{b}
\end{array}\right\}, \quad \text { and } \quad u_{a e}=\left\{\begin{array}{c}
\chi_{b} \\
\dot{\chi}_{b}
\end{array}\right\} .
$$

where $\Gamma_{b}$ and $\Gamma_{w}$ are bound (surface) and wake circulation strengths, respectively, and $\chi_{b}$ is the aerodynamic grid. This linearized state-space UVLM provides a compact and efficient tool, of the same fidelity that frequency-domain doublet-lattice (DLM) solvers, but without some of its restrictions: the wake can be nonplanar, flow tangency is imposed on the statically deformed geometry, and in-plane deformations are captured. Besides, it enables rigid-body motions and elastic deformations to be incorporated in a unified monolithic framework.

\section{III.B. Monolithic description of linearized flexible-aircraft dynamics}

The flexible-body equations are expressed in continuous time, whereas the linear UVLM is written in discrete time. Hence, temporal discretization of the beam equations is required for the fluid/structure coupling. This was performed through a standard Newmark- $\beta$ discretization. This leads to a seamless discrete-time statespace formulation, which for the homogeneous problem has the form ${ }^{34}$

$$
E_{\text {sys }} \Delta x^{n+1}=A_{\text {sys }} \Delta x^{n},
$$

where the state vector that completely determines the linear system is

$$
x=\left[x_{a e}^{T}\left|x_{S}^{T}\right| x_{R}^{T}\right]^{T}=\left[\Gamma_{b}^{T} \Gamma_{w}^{T} \dot{\Gamma}_{b}^{T}\left|\eta^{T} \dot{\eta}^{T}\right| \beta^{T} \Theta^{T}\right]^{T},
$$

where $\Theta$ are Euler angles, used to define the orientation of the body-fixed frame. The coupling of the flexibleaircraft equations is monolithic, since the interface between aerodynamics and flexible body-dynamics is determined analytically. The linearization might take place about a highly deformed configuration, and that although the wake is frozen, interference-induced unsteady downwash effects are modeled.

Eq. (12) defines a discrete-time generalized eigenvalue problem to determine the dynamic stability of the vehicle. Note that this is done on the original discretization of the structural and aerodynamic problems (the nodal variables on the finite-element and the circulations on the aerodynamic lattice), without any need to pre-compute aerodynamic forces in the frequency domain or prior projection on the natural modes of the structure (the latter however can be included as shown in section II.C). The system is linear and one could use linear techniques for model reduction at this stage, ${ }^{1}$ but this will not be investigated here. Note also that the formulation lends itself to time marching, gust response and control synthesis. ${ }^{33}$ Finally, note that the geometrically-nonlinear beam model has been used to introduce nonlinear static equilibrium conditions, but the analysis could be also based on the linear normal modes of the structure. 


\section{III.C. Monolithic description of nonlinear flexible-aircraft dynamics with small deformations}

Finally, we can also extend the seamless discrete-time state-space formulation given in Eq. (12) to situations with large rigid-body vehicle rotations, but with still small elastic deformations. As before, this is defined about a geometrically-nonlinear static equilibrium. Taking the equations of motion in modal basis, Eqs. (8), as starting point, and defining $q^{\top}=\left[p^{\top} \dot{p}^{\top}\right]$, the structural equations can be written in compact form as

$$
\begin{aligned}
& \dot{q}=f_{S S}(\beta) q+f_{S R}(\beta)+b_{S} y_{a e}, \\
& \dot{\beta}=f_{R S}(\beta) q+f_{R R}(\beta)+b_{R} y_{a e},
\end{aligned}
$$

with quadratic functions in $\beta$ and with $y_{a e}$ the forces in the aerodynamic panels computed as the output of Eq. (10). These equations are now easily written in discrete-time form, and assembled with the aerodynamic equations (10) to provide the tightly-coupled equations of motion with nonlinear rigid-body dynamics. In the actual implementation in this work, the second-order equations (8) were originally discretized in time using Newmark- $\beta$ and then directly coupled with the aerodynamics.

\section{Numerical Studies}

We have implemented this formulation in a framework for Simulation of High-Aspect-Ratio Planes (SHARP). Previous work ${ }^{20,31-33}$ has included extensive verification studies of the framework for nonlinear static and dynamic solutions of flexible multi-beam configurations including aerodynamics. In particular, the linear-flexible/nonlinear-rigid dynamics formulation, outlined in Section II.B, was exercised in Hesse and Palacios $^{32}$ on configurations with prescribed loads. Those results served to evaluate the limits of applicability of the mean-axes approximation for a multi-beam problem in spiral motion.

In this work, we firstly return to the spiral problem of the previously analyzed multi-beam configuration ${ }^{32}$ to gain a deeper understanding of the effect of gyroscopic motion on the mean-axes approximation. In the second part, a representative flexible HALE aircraft is introduced to exercise the linear-flexible/nonlinear-rigid dynamics formulation coupled with the nonlinear aerodynamics solver. First, the stability characteristics of the aircraft are presented which are then demonstrated on the open-loop response around a perturbed trim configuration. This problem serves to analyse the effect of geometric nonlinearity on the dynamic response of the vehicle which will be finalized with a comparison to the mean-axes approximation for relatively stiff aircraft.

The mean-axes constraint is naturally enforced by projecting the linear EoM onto free-free modes. ${ }^{39}$ Here, we obtain the free-free modes in the classical sense by solving the eigenvalue problem given by the unconstrained, undamped elastic EoM as, $\mathcal{M}^{S S} \ddot{\eta}^{f}+\mathcal{K}_{\text {stif }}^{S} \eta^{f}=0$, where $\eta^{f}$ is the vector of elastic DoF without enforcing the boundary condition of the clamped node at the origin of frame $A$. The corresponding stiffness matrix, $\mathcal{K}_{\text {stif }}^{S}$, is obtained through linearization of the elastic forces, $Q_{s t i f}^{S}$. The relative displacements of the flexible body can then be described in terms of modes shapes, $\Phi^{f}$, and generalized displacements, $p^{f}$, such that $\eta^{f}=\sum_{i} \Phi_{i}^{f} p_{i}^{f}$. Due to orthogonality of the free-free modes and if we neglect the contribution of elastic deformations to the gyroscopic forces in Eq. (3), then it is possible to solve the mean-axes EoM,

$$
\ddot{p}_{i}^{f}+\omega_{i}^{2} p_{i}^{f}=\left(\Phi_{i}^{f}\right)^{\top} Q_{e x t}^{f}(\eta, \beta, \zeta),
$$

separately from the nonlinear rigid-body dynamics EoM, extracted from Eq. (3), as

$$
\mathcal{M}^{R R}(\eta) \dot{\beta}+Q_{g y r}^{R}(\eta, \beta)=Q_{e x t}^{R}(\eta, \beta, \zeta),
$$

where $\omega_{i}$ is the vibration frequency of the $i$ th mode. However, the resulting set of EoM can still be coupled if the external forces depend on the rigid-body velocities, $\beta$, and orientation, $\zeta$, respectively.

\section{IV.A. Assessment of Mean-Axes Constraint on a Flexible Multi-Beam (FMB) Configuration}

In this section, we return to the flexible multi-beam configuration (FMB) in spiral motion, previously analyzed in Hesse and Palacios, ${ }^{32}$ to investigate the effect of the gyroscopic forces on the validity of the mean-axes approach by varying the radius of the prescribed spiral trajectory and hence the magnitude of the resulting gyroscopic terms. The geometry, material properties and load history of the FMB are defined in Fig. 2. A 
set of follower forces and moments is now acting at the CM of the initially undeformed geometry, which is rigidly-linked to the frame at point $P_{G}$. This implies that, under deformation of the structure, the origin of loading may not remain at the CM. The follower loading is applied as a ramp with time $t$ such that the FMB follows a circular rigid-body motion with radius $R_{S}=80 \mathrm{~m}$, where $m$ is the total mass of the frame and $J_{z}$ is the (constant) moment of inertia around the $z$ axis at the reference configuration. An additional dead load is applied at an offset at point $P_{G}$, shown in Fig. 2, to stabilize the structure, as the FMB tends to diverge from the spiral path under deformation. The parameter $p_{R}$ is introduced here to increase the radius of the prescribed spiral motion. A mesh size of 602 -noded elements and a time step of $\Delta t=0.1 \mathrm{~s}$ was found to be sufficient to capture the predominantly rigid-body dynamics of the problem simulated for $80 \mathrm{~s}$. The Newmark constant-acceleration scheme was modified as in Géradin and Rixen ${ }^{40}$ to include numerical damping of 0.01 that filters some high-frequency oscillations.

Material properties:

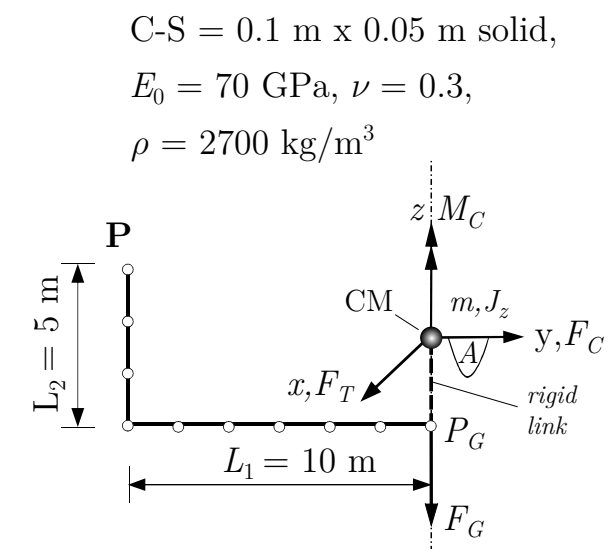

Spiral radius:

$$
R_{S}=80 \mathrm{~m} \cdot p_{R}
$$

Follower loads:

$$
\begin{aligned}
F_{T}(t) & =5 \mathrm{~N} / \mathrm{s} \cdot t \\
M_{C}(t) & =m F_{T}(t) /\left(J_{z} R_{S}\right) \\
F_{C}(t) & =m v_{A y}(t)^{2} / R_{S}
\end{aligned}
$$

Dead loads:

$$
F_{G}(t)=2 \mathrm{~N} / \mathrm{s} \cdot t
$$

Figure 2: Definition of the FMB problem.

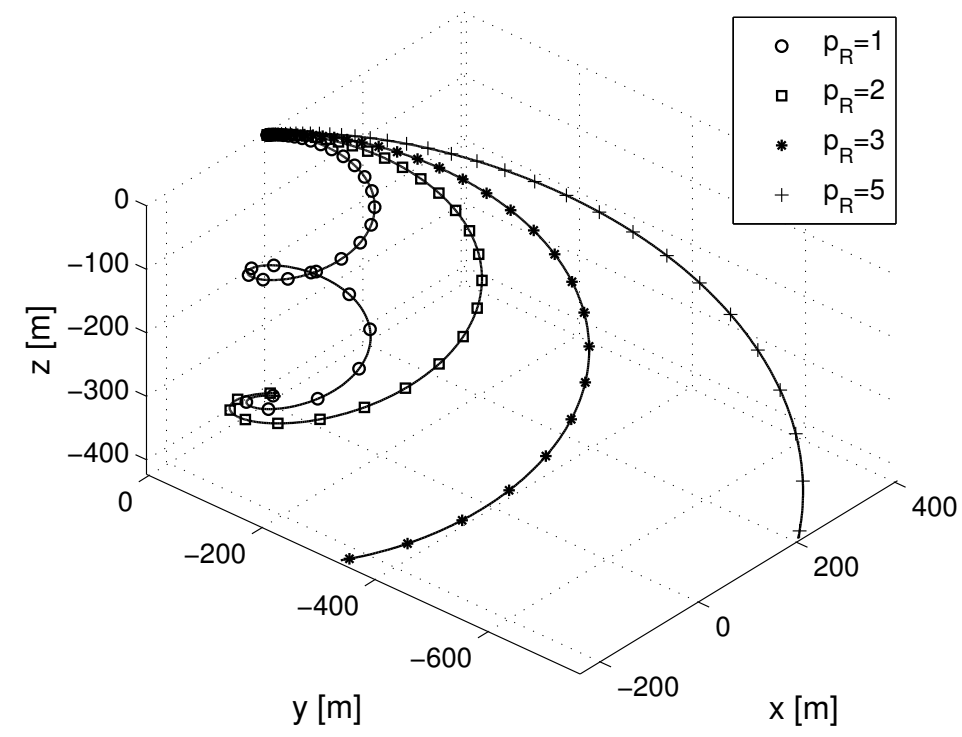

Figure 3: Trajectory of the origin of the body-fixed frame for the FMB problem and different radii. Displacement in the $z$-direction is equal in all cases. $[\sigma=50, \Delta t=0.1 \mathrm{~s}$ and 602 -noded elements]

The stiffness parameter $\sigma$ is used in this problem to vary the stiffness of the structure. Previous work ${ }^{32}$ established the effect of flexibility and geometric nonlinearity on the spiral response. It was shown for $p_{R}=1$ 
that the elastic deformations become linear for $\sigma \geq 10$ (with relative displacements of less than $4 \%$ of arm length $L_{2}$ ) and the overall motion converges to the rigid case for $\sigma \geq 50$ (less than $1 \%$ relative displacements). Within this stiff range of $\sigma \geq 50$ the relative error norm of the mean-axes assumption to predict the tip deformations was found to converge to a constant value of about $12 \%$ for $p_{R}=1$. This range of $\sigma$ values will be the center of focus in this study to investigate the effect of gyroscopic motion on the relative error of the mean-axes constraint.

The overall motion of the centroid for $\sigma=50$ is shown in Fig. 3 for a range of parameters $p_{R}$. The corresponding rigid-body velocities producing the spiral motions are presented in Fig. 4 for $p_{R}=1$ and $p_{R}=5$. For very stiff problems $(\sigma \geq 50)$ the prescribed forces result in zero angular velocity components around $x$ and $y$ axes (not shown in Fig. 4) and the maximum $\omega_{G z}$ component at $t_{e}=80 \mathrm{~s}$ decreases exponentially for increasing $p_{R}$. The translational velocity components $v_{G x}$ and $v_{G y}$ are oscillatory due to the circular motion and exhibit a constant $L^{2}$ norm at $t_{e}=80 \mathrm{~s}$ for varying radii.

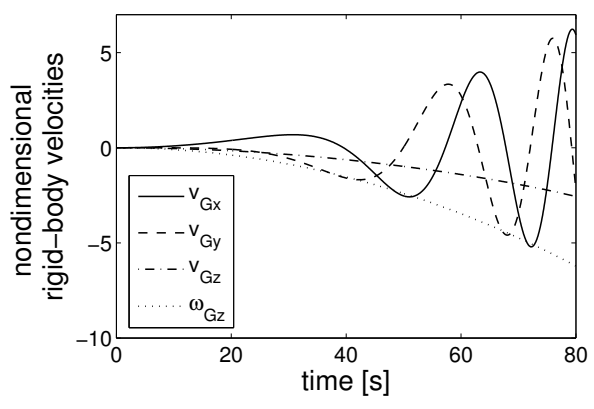

(a) $p_{R}=1$

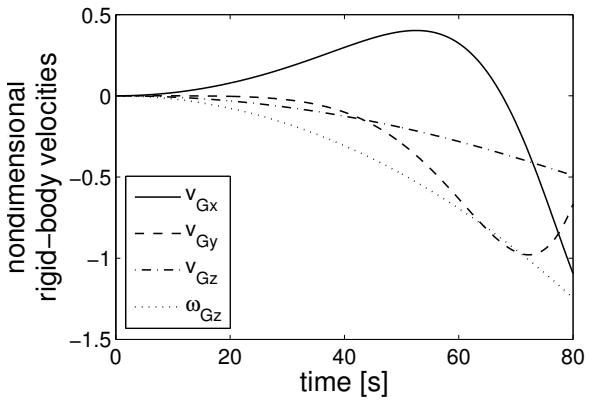

(b) $p_{R}=5$

Figure 4: Rigid-body velocity components of the FMB spiral problem for different radii nondimensionalized with $2 \pi p_{R} / t_{e}$ (translational components) and $2 \pi / t_{e}$ (rotational components) with $t_{e}=80 \mathrm{~s} . \quad[\sigma=50$, $\Delta t=0.1 \mathrm{~s}$ and 60 2-noded elements]

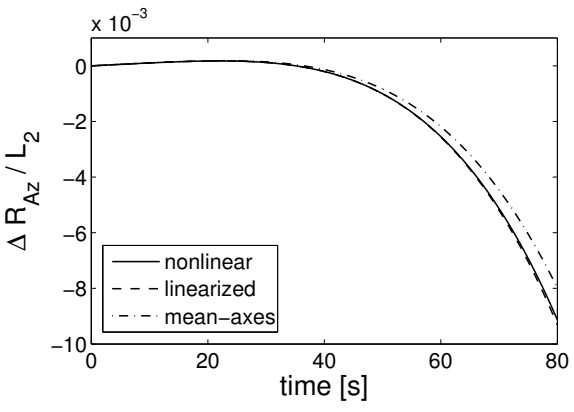

(a) $p_{R}=1$

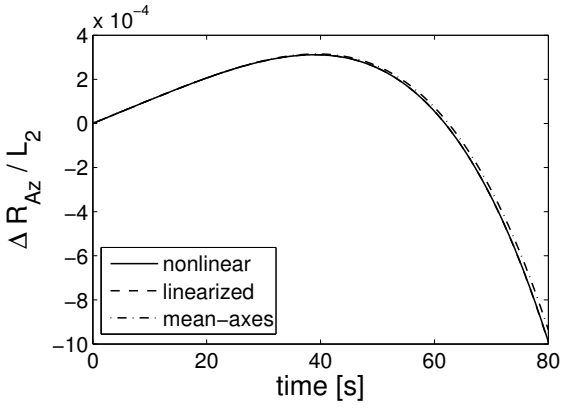

(b) $p_{R}=5$

Figure 5: Vertical displacement of the FMB free end in the spiral problem and for different radii and models. $[\sigma=50, \Delta t=0.1 \mathrm{~s}$ and 602 -noded elements $]$

The resulting normalized tip displacements due to centrifugal forces, shown in Fig. 5 for $\sigma=50$ and $p_{R}=1,5$, remain below $1 \%$ relative to the arm length $L_{2}$ defined in Fig. 2 . It was shown in Hesse and Palacios ${ }^{32}$ that the linear-flexible/nonlinear-rigid dynamics formulation tends to over-predict the elastic response for very flexible cases $(\sigma \leq 10)$ as the hardening effect due to large deformations is not captured. Comparison between fully-nonlinear (Eq. (3)), linearized (Eq. (5)) and mean-axes solutions (Eq. (15)) in Fig. 5 demonstrates that the elastic response is linear for chosen range of $\sigma$ values. However, it is clear that application of the mean-axes constraint results in smaller deflections, which converge to the nonlinear solution for larger radii and hence smaller angular velocities.

Figure 6a shows the maximum relative error norm of the mean-axes approach in predicting the deflection of point $\mathrm{P}$ for a range of $\sigma$ values and radii. For all cases presented here the maximum always occurs at $t_{e}=80 \mathrm{~s}$ when the tip deflection is greatest. It is obvious that neglecting the coupling terms results in 
a constant error for varying material stiffness, but decreases exponentially with increasing radius. This behavior is illustrated in detail in Fig. 6b which shows the correlation between the relative error of the right tip deflection and its components with the spiral radius for $\sigma=50$. Whereas the error in out-of-plane deformations, $\max \left(\Delta R_{A x} / L_{2}\right)$, is negligible, errors due to anti-symmetric bending contribute mostly to the overall relative error norm.

From Figs. 4 and 6 it is evident that there is a direct correlation between the angular velocity component $\omega_{G z}$, which decreases exponentially with increasing $p_{R}$, and the relative error of the mean-axes constraint to predict the elastic response. As discussed above, the contribution of the gyroscopic forces to damping and stiffness matrices, $\overline{\mathcal{C}}^{S S}, \overline{\mathcal{C}}^{S R}$ and $\overline{\mathcal{K}}_{\text {gyr }}^{S}$, are neglected under the mean-axes assumption in order to decouple the modal system equations. The contribution of the gyroscopic forces in this problem originates from the angular velocity $\omega_{G z}$ alone, as the norm of the translational velocity components is constant for varying radii.

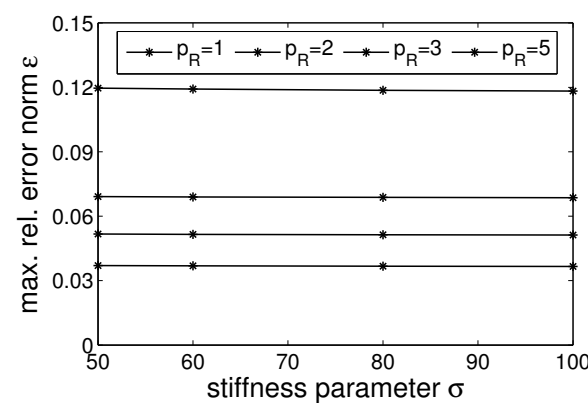

(a) $\max \left(\Delta R_{A} / L_{2}\right)$ for varying $\sigma$ and $p_{R}$.

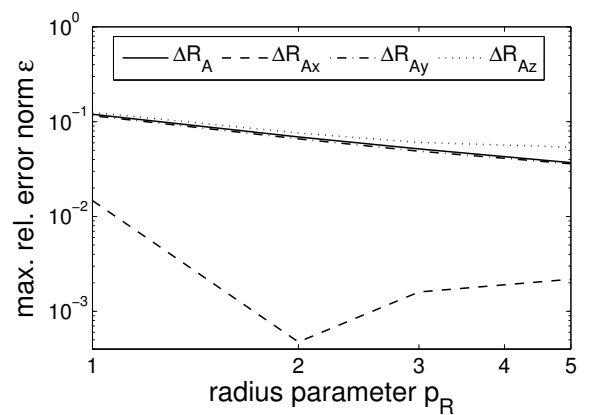

(b) $\max \left(\Delta R_{A} / L_{2}\right)$ and $\max \left(\Delta R_{A x, y, z} / L_{2}\right)$ for $\sigma=50$ and different radii.

Figure 6: Maximum relative error norms of tip displacement at Point $\mathrm{P}$ for the FMB spiral problem comparing the mean-axes approximations to the nonlinear solution for different radii. $[\Delta t=0.1 \mathrm{~s}$ and 60 2-noded elements]

\section{IV.B. Effect of Mean-Axes Constraint on Flexible Aircraft Flight Dynamics}

A similar study can be carried out on a full aircraft configuration. The representative HALE aircraft used in this section is defined in Fig. 7 and Table 2. It is loosely based on the design by Patil et al., ${ }^{12}$ but it includes winglets and the payload of $50 \mathrm{~kg}$ was moved to the front of the fuselage to guarantee static pitch stability. ${ }^{31}$ The vehicle consists of large aspect ratio flexible wings, a rigid fuselage and a rigid empennage. The overall semi-span of the main wing is $B=16 \mathrm{~m}$, which encompasses rigidly-linked dihedral members at both ends at an angle of $20 \mathrm{deg}$. The ratio of the span of each dihedral member over the total semi-span of the main wing, $B$, is defined as $p_{d i h}$ which will be used in this study to vary the size of the dihedral members. The horizontal stabilizer and the vertical fin are modeled as lifting surfaces, and they include an elevator modeled as a quarter-chord-length control surfaces. The fuselage, in turn, is represented by a non-lifting beam, and it is inclined so that the empennage is raised by $1.25 \mathrm{~m}$ with respect to the main wing to avoid wake-tail collisions.

The aircraft is powered by two massless propellers, which are modeled as point forces rigidly linked to the wing. The mass per unit length of the fuselage is the same as that of the horizontal and vertical tail planes, and thus the total mass of this aircraft, including payload and structural mass, is $75.4 \mathrm{~kg}$. It is assumed that the aircraft flies with $V_{\infty}=30 \mathrm{~m} / \mathrm{s}$ at an altitude of $20 \mathrm{~km}$, where the density is $\rho_{\infty}=0.0899$ $\mathrm{kg} / \mathrm{m}^{3}$. The relevant properties of the members are listed in Table 2. The stiffness parameter $\sigma$ will be used in the following studies to vary the stiffness properties of the main wing (decreasing $\sigma$ values result in a more flexible wing).

To study the stability characteristics and open-loop response of the HALE aircraft in the subsequent sections, the vehicle is trimmed for steady level flight through three inputs, namely angle of attack, AoA, elevator deflection, $\delta_{e}$, and thrust per propeller, $T$. The trimming routine in SHARP is a tightly-coupled process which is described in detail in Murua et al. ${ }^{34}$ The trim characteristics of the HALE aircraft are 
(a)

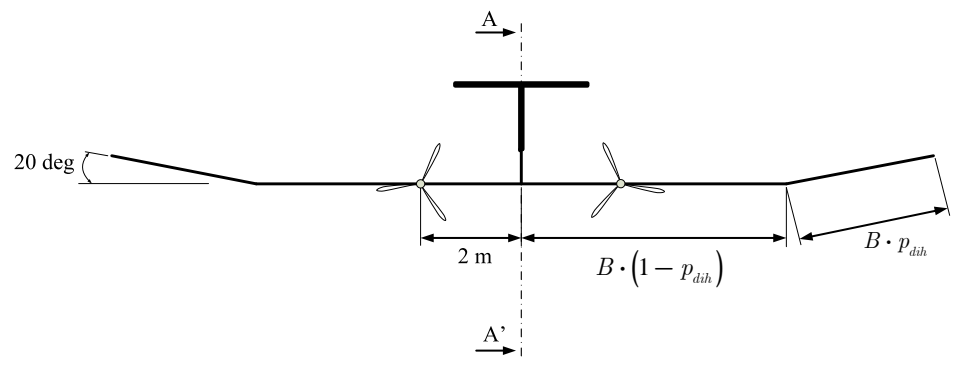

(b)

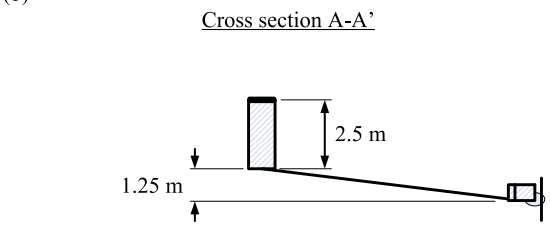

(d)

(c)

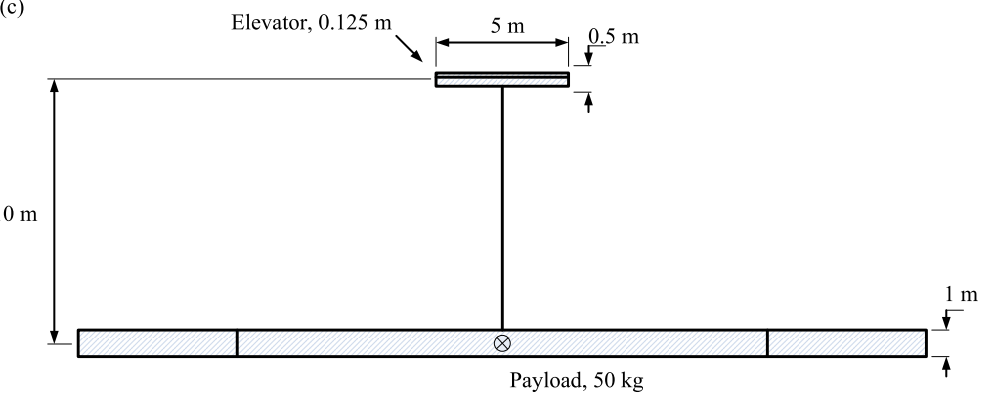

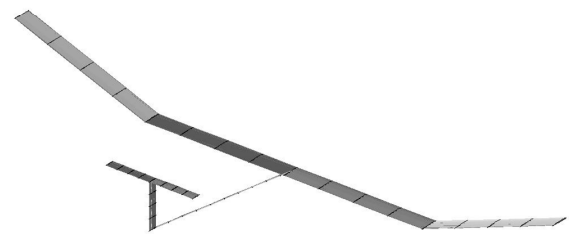

Figure 7: Undeformed HALE aircraft geometry: (a) front view, (b) cross-section, (c) top view, and (d) 3D view showing the aerodynamic lattice.

Table 2: HALE model aircraft properties.

\begin{tabular}{lll}
\hline \hline & Main wing & Tail plane \\
\hline Chord & $1 \mathrm{~m}$ & $0.5 \mathrm{~m}$ \\
Total semi-span, $B$ & $16 \mathrm{~m}$ & $2.5 \mathrm{~m}$ \\
Elastic axis & $50 \%$ chord & $50 \%$ chord \\
Center of gravity & $50 \%$ chord & $50 \% \mathrm{chord}$ \\
Mass per unit length & $0.75 \mathrm{~kg} / \mathrm{m}$ & $0.08 \mathrm{~kg} / \mathrm{m}$ \\
Moment of inertia & $0.1 \mathrm{~kg} \cdot \mathrm{m}$ & $0.01 \mathrm{~kg} \cdot \mathrm{m}$ \\
Torsional stiffness & $1 \sigma \times 10^{4} \mathrm{~N} \cdot \mathrm{m}^{2}$ & $\infty$ \\
Bending stiffness & $2 \sigma \times 10^{4} \mathrm{~N} \cdot \mathrm{m}^{2}$ & $\infty$ \\
In-plane bending stiffness & $4 \sigma \times 10^{6} \mathrm{~N} \cdot \mathrm{m}^{2}$ & $\infty$ \\
\hline \hline
\end{tabular}

presented in Fig. 8 for varying dihedral member span ratio, $0 \leq p_{\text {dih }} \leq 1 / 2$, and stiffness parameter of the main wing, $5000 \leq \sigma \leq 1.5$. The tip deflection of the main wing, non-dimensionalized with the overall semi-span of the main wing, $B$, is also included in Fig. 8. It can be seen that with increasing span ratio of the dihedral members, $p_{d i h}$, the loss of vertical forces is compensated by a larger angle of attack and elevator deflection. The wing tip deflection initially decreases for increasing values of $p_{d i h}$ due to the decrease in bending moment from the dihedral members, as the lift vector is pointing inwards. However, as the angle of attack increases with dihedral span, the additional lifting forces acting perpendicular to the dihedral members counteract the drop in bending moment and result in larger tip deflections. This is evident in Fig. 8 for $p_{d i h}=1 / 2$. For the most flexible case $(\sigma=1.5)$ analyzed in this section the trim deformations reach around $28 \%$ of the wing semi-span.

\section{IV.B.1. Linear Stability Analysis}

Firstly, the stability characteristics of the presented aircraft are analyzed using the stability tool in SHARP. ${ }^{31,34}$ The solution process can be summarized as follows: the equilibrium trim condition is computed according 


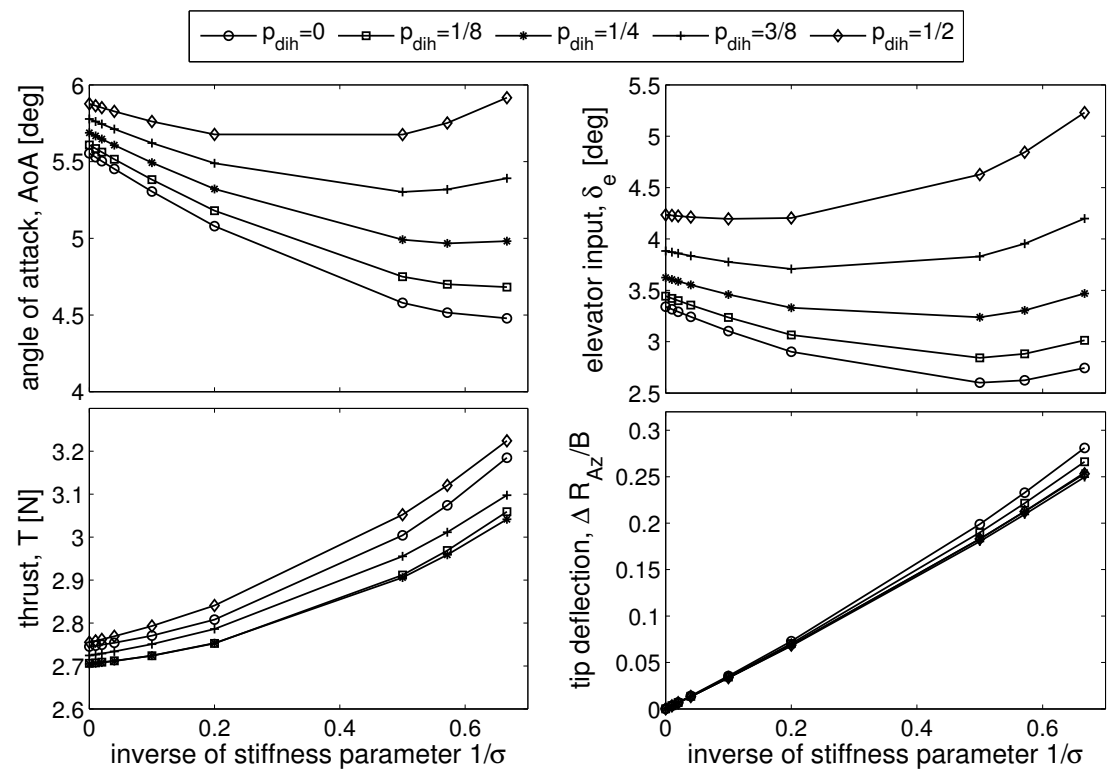

Figure 8: Trim characteristics of the HALE aircraft as a function of wing flexibility, $\sigma$, and dihedral member span ratio, $p_{d i h}$, with the trim inputs in angle of attack, elevator deflection, and thrust per propeller and the corresponding non-dimensional vertical tip deflection of the main wing.

to the trim inputs presented in Fig. 8 and a linear stability analysis is carried out about the deformed trim configuration. This is accomplished by solving directly the generalized eigenvalue problem posed by the linear aeroelastic system coupled with the flight dynamics of the flexible aircraft, given by Eq. (12). Note that in this case pre-computing frequency-domain aerodynamics is not necessary, neither is modal projection.

Fig. 9 presents the root loci as a function of the wing stiffness, $\sigma$, for a dihedral ratio of $p_{d i h}$. Note that the eigenvalue analysis defined by Eq. (12) yields as many roots as states has the problem, but only the most dominant ones are presented. These eigenvalues are shown in the plots, identifying the corresponding mode. The latter is determined from the associated eigenvector, also obtained from the solution of Eq. (12). The first few modes of the flexible vehicle are designated by the names of their rigid-aircraft counterparts, e.g., phugoid, short period, etc., although they also include vehicle deformations. The following unevenly-spaced values of sigma have been considered: $\sigma=\left[\begin{array}{lllllllllll}1.5 & 1.75 & 2 & 5 & 10 & 25 & 50 & 100 & 500 & 1000 & 5000\end{array}\right]$. The two extreme cases are representative of a very flexible wing, $\sigma=1.5$, and a rigid one, $\sigma=5000$. Note that the solution of Eq. (12) are discrete-time eigenvalues, but they have been converted to continuous time for an easier interpretation.

Two classical flight-dynamics lateral modes can be observed in Fig. 9(a), namely the oscillatory Dutch roll and the non-oscillatory spiral divergence. As flexibility increases, the Dutch roll becomes less stable. While it remains stable across the range of stiffness values considered, it illustrates the detrimental impact flexibility might have on the handling qualities of the vehicle. In contrast, the spiral mode presents the opposite trend: the stability margin substantially increases as the wing stiffness is reduced. This is due to the larger static deformations of the wing at trim conditions, which offer a higher resilience against the spiral mode.

Fig. 9(b) zooms further in the root loci in order to visualize the phugoid, another of the characteristic flight-dynamics modes. This longitudinal mode features very low frequency pitching and plunging motions of the aircraft, leading to a trajectory of a nearly constant angle of incidence. For a rigid aircraft it is generally a lightly damped mode. As flexibility is introduced, the mode epitomizes the coupling of elastic and rigid-body degrees of freedom: as the aircraft pitches up and climbs first, the wings undergo a downward flapping-like motion. As the vehicles pitches down and descends, the wing bends upwards, reaching the maximum tip deflections before the cycle is finished. Whereas rigid and stiff aircraft present a stable phugoid, the damping drops, approaching the neutral boundary and eventually becoming unstable as the wing flexibility increases. 

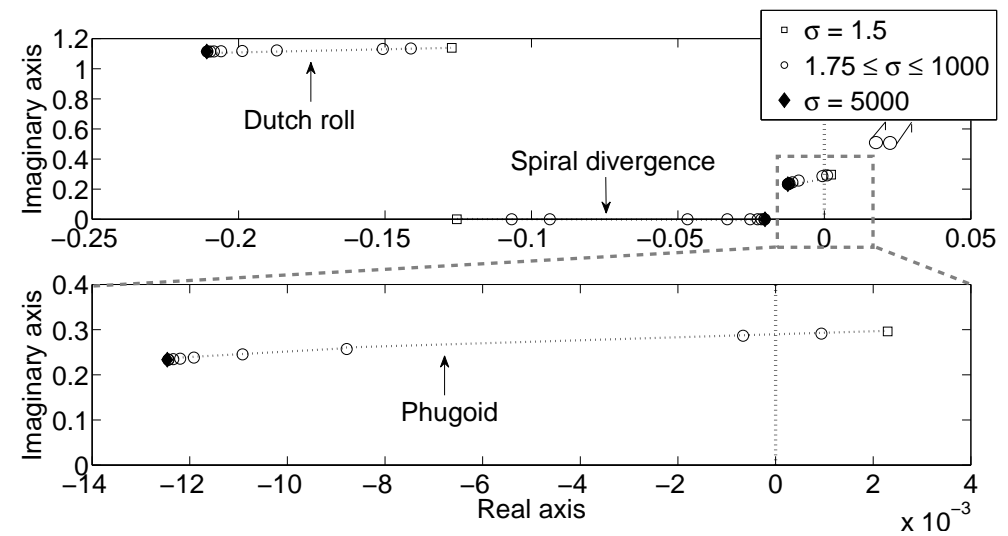

Figure 9: Root loci of the eigenvalue analysis for $p_{d i h}=2$, with the stiffness of the main wing as parameter: (a) dominant roots, and (b) magnified section showing the roots nearest the origin.

Note finally, that other modes, such as the short period and roll subsidence, along with many aeroelastic modes are predicted by this stability analysis, but fall beyond the figure boundaries.

In order to conclude this parametric study on the stability characteristics of the aircraft, the effect of the outboard dihedral will be investigated. Fig. 10 shows the root loci for both rigid $(\sigma=5000)$ and flexible $(\sigma=1.5)$ aircraft. The same dominant modes appear in this case as well: Dutch roll, spiral divergence, and phugoid.

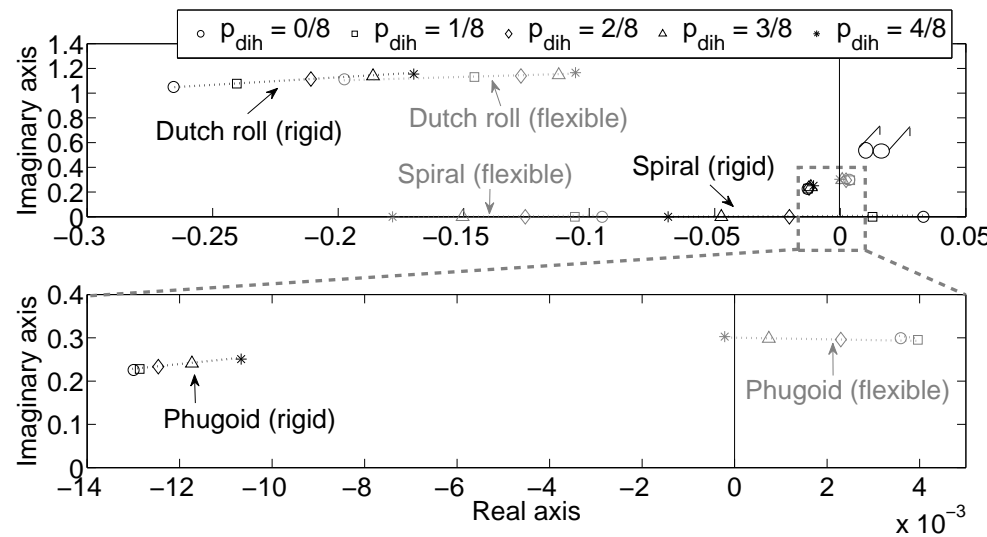

Figure 10: Root loci of the eigenvalue analysis for $\sigma=1.5$, with the dihedral ratio as parameter: (a) dominant roots, and (b) magnified section showing the roots nearest the origin.

Fig. 10(a) shows that an increase of the dihedral ratio leads to a less stable Dutch roll, while it increases the resistance against spiral divergence. This coincides with the trends observed in Fig. 9(a) with increased flexibility. That is, static deformations and dihedral ratio have the same effect in the lateral modes, increasing the stability margin of the spiral, and reducing it for the Dutch roll. As the dihedral ratio is varied, these trends are consistent for both the rigid and flexible vehicles. As expected, wings with very small or no dihedral lead to unstable spiral modes.

Fig. 10(b) focuses on the region closer to the origin in order to expose the characteristics of the phugoid mode. Interestingly, the trends are opposite for the rigid and flexible vehicles: the increase of dihedral ratio leads to a more stable phugoid in the flexible aircraft (eventually stable for $p_{d i h}=4 / 8$ ), but the damping of the rigid phugoid decreases with $p_{d i h}$. Two main factors seem to play a role in stability as flexibility is introduced: on the one hand, the shape of the deformed wing at static equilibrium; on the other, the aeroelastic and flight-dynamics coupling. Whereas the behavior of the spiral mode is mostly governed by the shape of the wing (through dihedral or static deformations), the phugoid mode of the flexible vehicle is likely to be driven by the overlap of structural and rigid-body frequencies. 


\section{IV.B.2. Open-Loop response of HALE aircraft subjected to initial roll perturbation}

To investigate the effect of consistent linearization of the structural DoF, we analyze the dynamic behavior of the presented HALE aircraft subject to an initial perturbation away from the original trim configuration. The perturbation is introduced in the form of an initial roll angle of $10 \mathrm{deg}$. The static equilibrium is computed for this new flight condition using the nonlinear static aeroelastic module in SHARP with the trim inputs presented in Fig. 8, and the aircraft is subsequently released to compute its open-loop response for $50 \mathrm{~s}$ using a time step of $d t=0.05 \mathrm{s.}{ }^{\mathrm{b}}$

The parameters $\sigma$ and $p_{d i h}$ are used in this section to explore the effect of flexibility and initial wing dihedral ratio on the nonlinear flight dynamic response which are compared to the linear stability characteristics presented in Figs. 9-10. The geometrically-nonlinear flexible-body dynamics description coupled with the nonlinear aerodynamics will provide a reference solution. Secondly, the question arises if a similar response can be computed using reduced-order models to describe the structural dynamics of the aircraft such as the modal form of the nonlinear flexible-body EoM presented in Section II.C. This will enable us to subsequently examine the effect of decoupling the elastic and rigid-body dynamics, as done in the mean-axes approximation. In order to isolate the effect of linearization of the structural DoF only, the aerodynamics were computed using the nonlinear description of the UVLM for all results presented here.

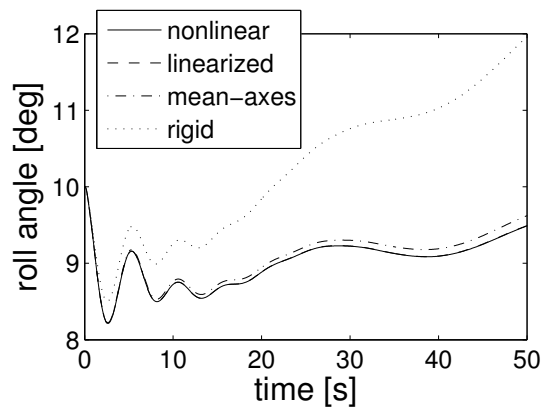

(a) $\sigma=25$

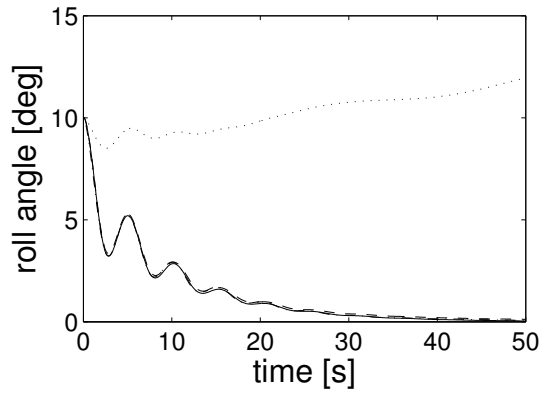

(b) $\sigma=1.5$

Figure 11: Roll response of the HALE aircraft for dihedral member ratio $p_{\text {dih }}=1 / 4$ and different stiffness parameter $\sigma$. The dotted curve refers to the rigid aircraft response.

As one would expect from the discussion of the stability characteristics in the previous section, we found that the rigid aircraft subjected to an initial roll angle returns to a trim condition for dihedral ratios which yield a stable spiral mode $\left(p_{d i h}>1 / 4\right)$ and diverges in the unstable cases. The configuration at the transition of the spiral stability $\left(p_{d i h}=1 / 4\right)$ will be the most interesting configuration to analyze here and Fig. 11 compares the roll response for the rigid aircraft with two different flexible configurations which correspond to equilibrium tip deflections of $1.2 \%(\sigma=25)$ and $23.5 \%(\sigma=1.5)$ of the wing semi-span. Even though Fig. 9 shows that for $p_{d i h}=1 / 4$ the rigid aircraft is marginally stable in the linear regime, which results in an initial restoring roll moment, the aircraft eventually becomes unstable. The effect of increasing flexibility on the spiral stability is evident in the case of $\sigma=1.5$ for which the aircraft stabilizes to a new trim condition after $t=50 \mathrm{~s}$. Despite initial oscillations due to large initial roll moments, the roll angle decays exponentially with a damping ratio in the same order of magnitude as the value presented in Fig. 9.

The roll angle at $t=50 \mathrm{~s}$ is used in Fig. 12 as a measure to compare the flight dynamic response for varying stiffness parameter and dihedral ratio. These results reflect the stability properties of the aircraft and show that independent of the initial dihedral ratio the flexible vehicle returns to a stable trim condition for $\sigma \leq 5$. This effect on the roll response can have a large impact on the trajectory of the HALE aircraft, which is presented in Fig. 13 for the two different dihedral ratios $p_{d i h}=1 / 8$ and $1 / 2$ for varying stiffness of the main wing. The flight path is presented only in the $x-y$ plane since the altitude drop for this comparatively light aircraft is negligible compared to forward and lateral motion. The prescribed initial roll angle of $10 \mathrm{deg}$ results in a circular trajectory of the aircraft with radii proportional to the spiral stability which increases with flexibility and dihedral ratio.

\footnotetext{
${ }^{\mathrm{b}} \mathrm{A}$ convergence study showed that this large time step has a damping effect on the flight dynamic response, but the overall behavior is captured well. For the purpose of the parametric study in this paper this time step was justified, but further work is required using a refined mesh and time step.
} 


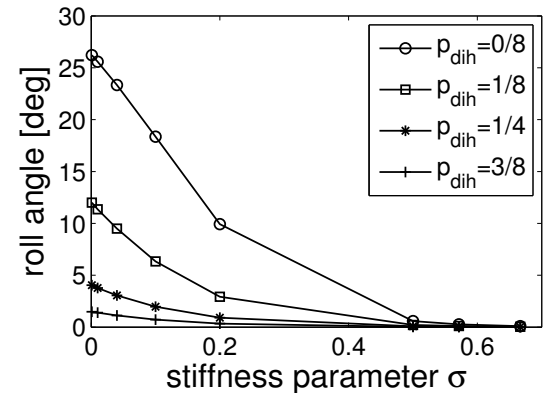

(a)

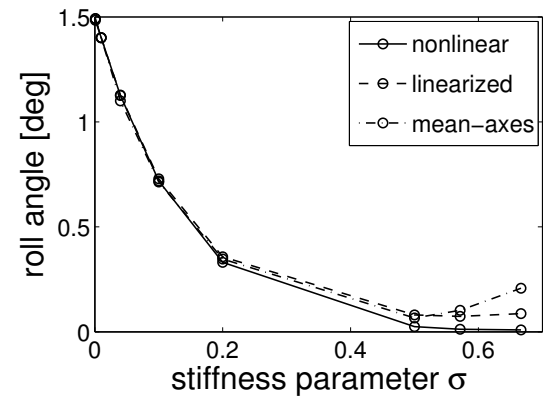

(b)

Figure 12: Roll angle of the HALE aircraft at $t=50 \mathrm{~s}$ for varying stiffness parameter $\sigma$ and (a) nonlinear solution with varying dihedral ratio $p_{d i h}$, (b) different formulations and a fixed dihedral ratio of $p_{d i h}=1 / 2$.

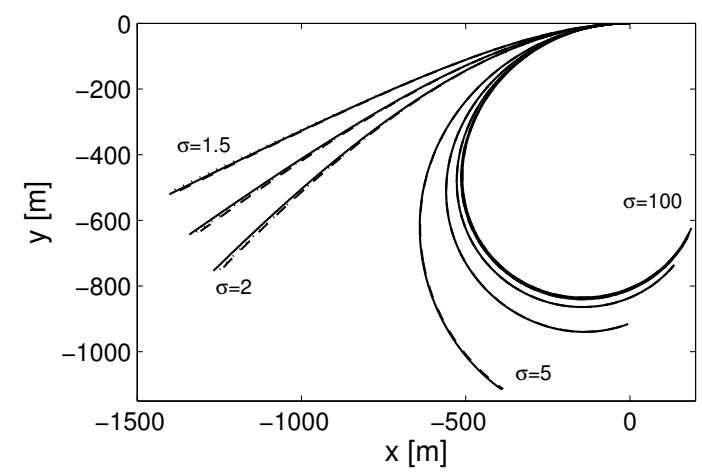

(a) $p_{\text {dih }}=1 / 8$

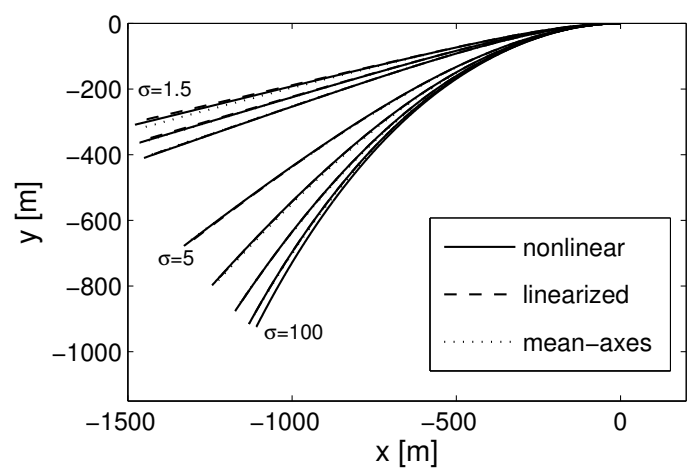

(b) $p_{d i h}=1 / 2$

Figure 13: Trajectory of the HALE aircraft in the $x-y$ plane for the different stiffness parameters $\sigma=$ $\left[\begin{array}{llllllll}1.5 & 1.75 & 2 & 5 & 10 & 25 & 100 & 1000\end{array}\right]$. The altitude drop is negligible compared to the forward motion.

The presented results demonstrate how significant the impact of the aircraft geometry and material stiffness is on the stability characteristics of the vehicle and subsequently the open-loop response due to roll perturbations. In order to compare the effect of linearization of the elastic DoF only, Figs. 11-13 also present the dynamic behavior obtained using the linear-flexible/nonlinear-rigid formulation, which suggests that the effect of geometric nonlinearity becomes dominant for $\sigma \geq 5$, but is negligible for stiffer aircraft. This is particularly evident in Fig. 12(b), which shows the comparison between nonlinear and linearized formulation to predict the roll angle at time $t=50 \mathrm{~s}$ for the dihedral ratio $p_{d i h}=1 / 2$. However, in the most flexible case $(\sigma=1.5)$ linearization results in an absolute error of less than $0.1 \mathrm{deg}$. The corresponding right tip deflection is presented in Fig. 14(b) for this configuration, which is the most extreme case in terms of displacements due to the large excitation of the initial roll moment. Whereas the most flexible case $(\sigma=1.5)$ exhibits equilibrium deformations of the right wing tip of $23.5 \%$ of the semi-span, the maximum amplitude in the transient around this equilibrium is below $2 \%$ of the semi-span. This shows that an increased flexibility of the main wing results in large, geometrically-nonlinear deformations at equilibrium, around which the elastic DoF can then be linearized to compute the transient, but the flight dynamics response is mostly affected by the initial equilibrium deformations.

At last, the response of the HALE aircraft due to the initial roll perturbation was computed using the mean-axes assumptions to decouple the flexible-body dynamics equations, but the aerodynamics are again computed on the actual instantaneous geometry. For small deformations this converges to the classical mean-axes approach commonly found in flight dynamic analysis. The mean-axes solution to the response of the HALE aircraft is included in Figs. 11-14 to compare with the fully-coupled nonlinear and the linearized solutions. It is obvious that for the open-loop response analyzed here the mean-axes approach can accurately predict the flight dynamic response, even for mildly flexible cases $(\sigma \geq 5)$. However, for more flexible configurations both simplified models diverge from the nonlinear solution as the large wing deformations 


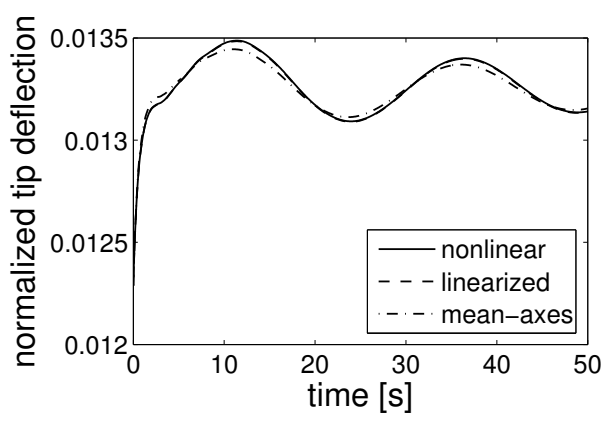

(a) $\sigma=25$

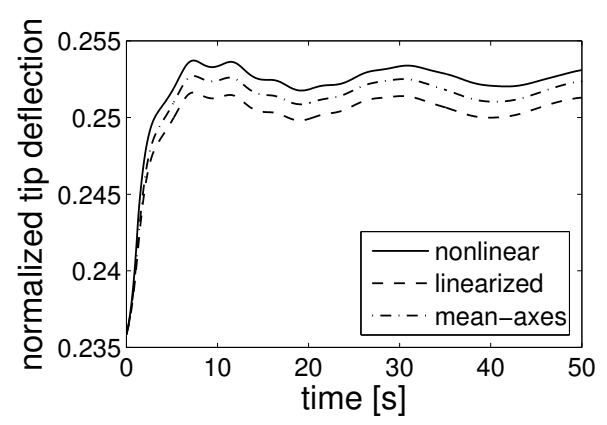

(b) $\sigma=1.5$

Figure 14: Vertical tip deflections of the right main wing normalized with the semi-span of $B=16 \mathrm{~s}$ for dihedral ratio of $p_{d i h}=1 / 2$.

begin to affect the flight behavior of the aircraft for $\sigma \leq 2$. This is evident in the comparison of the wing tip deflections in Fig. 14 for the most flexible case, for which both simplified models underpredict the elastic response. But even for the stiff case $(\sigma=25)$ corresponding to maximum deflections of $1.4 \%$ of the semispan, the mean-axes approach underpredicts the maximum amplitudes, which is comparable to the results found for the FMB problem in spiral motion.

In summary, this study demonstrated that the mean-axes approach can give a good estimate of the open-loop flight dynamic response, provided that the effect of elastic deformations in the transient is small compared to the (large) equilibrium deformations. However, it was also shown that the mean-axes assumption results in non-conservative prediction of the elastic deformations even for very stiff aircraft, which makes them less suitable for the prediction of dynamic loads.

\section{Conclusion}

This paper has presented the mathematical formulation of a coupled aeroelastic and flight dynamics framework with different model fidelities to compare the impact of linearization of the structural or aerodynamic degrees of freedom on the prediction of flight dynamics of flexible aircraft. This has relevance with respect to the design of next-generation passenger aircraft and medium-sized HALE vehicles, where the a small-sized model capable of capturing the flight behavior accurately is required for the design of control systems to optimize their performance and alleviate dynamic loads.

Starting point for this analysis was a displacement-based geometrically-nonlinear composite beam model which has been coupled with the Unsteady Vortex Lattice Method. Linearization of only the structural degrees of freedom resulted in flexible-body dynamics equations written in matrix form, in which the matrix coefficients have only a quadratic dependency on the body velocities, but the equations are linear in the elastic degrees of freedom. This feature was exploited by approximating the FEM discretization in terms a few vibration modes of the unconstrained structure. The resulting closed-form expressions for all the coefficients in the equations remain constant and only need to be computed once prior to a time-marching solution.

This modal form of the flexible-body equations of motion can capture all couplings between the nonlinear rigid-body dynamics and the linearized structural dynamics due to gyroscopic effects and lends itself to be integrated with the linearized state-space version of the Unsteady Vortex Lattice Method. This results in a novel formulation with a linear aeroelastic part of the system equations in modal space tightly coupled to the nonlinear flight dynamics of the vehicle and provides a reasonable alternative to the mean-axes approach which is typically used in flexible aircraft flight dynamics but neglects the influence of the gyroscopic forces on the structural dynamics.

Numerical results were presented in this paper first on a free-flying flexible multi-beam configurations without aerodynamics to investigate the effect of gyroscopic motion on the accuracy of the mean-axes assumption. It was shown that for a given problem the mean-axes constraint results in a constant relative error independent of the extent of deformations, but this error correlates directly to the angular velocity of the flexible body.

In the second part of this paper, a representative HALE configuration was introduced to exercise the 
linearized implementation of the unconstrained aeroelastic model on a flight dynamics problem with large lateral motions. A parameter study exposed the stability characteristics of the aircraft based on linear stability analyses and the open-loop response of the aircraft subject to an initial roll perturbation away from the trim condition. This study confirmed the dramatic effect of flexibility on the vehicle dynamics due to possibly large deformations at equilibrium condition, but also showed the negligible impact of geometric nonlinearity in the transient response, which confirms the motivation for further model reduction of the linear aeroelastic system with nonlinear flight dynamics. It was also shown that the mean-axes solution underpredicts the elastic deformations in this open-loop response even for stiff aircraft.

In summary, the linearized flexible-aircraft formulation provides a powerful tool to accurately describe the flight dynamics of current and next-generation aircraft concepts, which are likely to exhibit some extent of small wing deformations, and the model captures all coupling terms due to gyroscopic forces. As opposed to the mean-axes approach, this implies that the resulting linear elastic deformations can be captured accurately which is indeed critical for the prediction of surface loads, but the extra computational burden is minimal due to the modal projection of the structural degrees of freedom.

\section{Acknowledgements}

Joseba Murua gratefully acknowledges the financial support provided by the Department of Education, Universities and Research of the Basque Government. The work of Henrik Hesse is sponsored by the UK Engineering and Physical Sciences Research Council (EPSRC). This support is gratefully acknowledged.

\section{References}

\footnotetext{
${ }^{1}$ Cook, R. G., Palacios, R., and Goulart, P., "Robust Gust Alleviation and Stabilization of Very Flexible Aircraft," AIAA Journal, 2012, To appear.

${ }^{2}$ Milne, R. D., "Dynamics of the Deformable Plane," Tech. Rep. 3345, Her Majesty's Stationary Office, London, UK, Sept. 1962.

${ }^{3}$ Agrawal, O. P. and Shabana, A. A., "Application of deformable-body mean axis to flexible multibody system dynamics," Computer Methods in Applied Mechanics and Engineering, Vol. 56, No. 2, 1986, pp. 217-245.

${ }^{4}$ Friedmann, P. P., McNamara, J. J., Thuruthimattam, B. J., and Nydick, I., "Aeroelastic analysis of hypersonic vehicles," Journal of Fluids and Structures, Vol. 19, No. 5, 2004, pp. 681-712.

${ }^{5}$ Kier, T., Looye, G., Scharpenberg, M., and Reijerkerk, M., "Process, methods and tools for flexible aircraft flight dynamics model integration," International Forum on Aeroelasticity and Structural Dynamics, Stockholm, Sweden, June 2007.

${ }^{6}$ Albano, E. and Rodden, W. P., "A Doublet-Lattice Method for Calculating Lift Distributions on Oscillating Surfaces in Subsonic Flow," AIAA Journal, Vol. 7, No. 2, 1969, pp. 279-285.

${ }^{7}$ Karpel, M., "Design for Active Flutter Suppression and Gust Alleviation Using State Space Aeroelastic Modeling," Journal of Aircraft, Vol. 19, No. 3, 1982, pp. 221-227.

${ }^{8}$ Maute, K., Nikbay, M., and Farhat, C., "Coupled Analytical Sensitivity Analysis and Optimization of Three-Dimensional Nonlinear Aeroelastic Systems," AIAA Journal, Vol. 39, No. 11, 2001, pp. 2051-2061.

${ }^{9}$ Geuzaine, P., Brown, G., Harris, C., and Farhat, C., "Aeroelastic Dynamic Analysis of a Full F-16 Configuration for Various Flight Conditions," AIAA Journal, Vol. 41, No. 3, 2003, pp. 363-371.

${ }^{10}$ Garcia, J. A., "Numerical Investigation of Nonlinear Aeroelastic Effects on Flexible High-Aspect-Ratio Wings," Journal of Aircraft, Vol. 42, No. 4, July-August 2005, pp. 1025-1036.

${ }^{11}$ Patil, M. J., Hodges, D., and Cesnik, C., "Nonlinear Aeroelastic Analysis of Complete Aircraft in Subsonic Flow," Journal of Aircraft, Vol. 37, No. 5, 2000, pp. 753-760.

${ }^{12}$ Patil, M. J., Hodges, D. H., and Cesnik, C. E., "Nonlinear aeroelasticity and flight dynamics of high-altitude longendurance aircraft," Journal of Aircraft, Vol. 38, No. 1, 2001, pp. 88-94.

${ }^{13}$ Meirovitch, L. and Tuzcu, I., "Unified Theory for the Dynamics and Control of Maneuvering Flexible Aircraft," AIAA Journal, Vol. 42, No. 4, April 2004, pp. 714-727.

${ }^{14}$ Romeo, G., Frulla, G., Marzocca, P., and Tuzcu, I., "Non-Linear Aeroelastic Modeling and Experiments of Flexible Wings," $4^{\text {th }}$ AIAA/ASME/ASCE/AHS/ASC Structures, Structural Dynamics, and Materials Conference, AIAA 2006-2186, Newport, RI, USA, June 2006.

${ }^{15}$ Shearer, C. M. and Cesnik, C. E., "Nonlinear Flight Dynamics of Very Flexible Aircraft," Journal of Aircraft, Vol. 44, No. 5, 2007, pp. 1528-1545.

${ }^{16}$ Demasi, L. and Livne, E., "Dynamic Aeroelasticity of Structurally Nonlinear Configurations Using Linear Modally Reduced Aerodynamic Generalized Forces," AIAA Journal, Vol. 47, No. 1, January 2009, pp. 71-90.

${ }^{17} \mathrm{Su}, \mathrm{W}$. and Cesnik, C., "Nonlinear Aeroelasticity of a Very Flexible Blended-Wing-Body Aircraft," Journal of Aircraft, Vol. 47, No. 5, 2010, pp. 1539-1553.

${ }^{18}$ Zhao, Z. and Ren, G., "Multibody dynamic approach of flight dynamics and nonlinear aeroelasticity of flexible aircraft," AIAA Journal, Vol. 49, No. 1, January 2011, pp. 41-54.

${ }^{19} \mathrm{Katz}, \mathrm{J}$. and Plotkin, A., Low-Speed Aerodynamics, Cambridge Aerospace Series, Cambridge University Press, New York, NY, USA, 2nd ed., 2001.
} 
${ }^{20}$ Palacios, R., Murua, J., and Cook, R., "Structural and Aerodynamic Models in the Nonlinear Flight Dynamics of Very Flexible Aircraft," AIAA Journal, Vol. 48, No. 11, November 2010, pp. 2648-2559.

${ }^{21}$ Murua, J., Palacios, R., and Graham, J. M. R., "Assessment of Wake-Tail Interference Effects on the Dynamics of Flexible Aircraft," AIAA Journal, 2012, [Accepted for publication].

${ }^{22}$ Drela, M., "Integrated simulation model for preliminary aerodynamic, structural, and control-law design of aircraft," 40th AIAA/ASME/ASCE/AHS/ASC Structures, Structural Dynamics and Materials Conference, AIAA Paper 1999-1394, St. Louis, Missouri, USA, April 1999.

${ }^{23}$ Wang, Z., Chen, P. C., Liu, D. D., Mook, D. T., and Patil, M. J., "Time Domain Nonlinear Aeroelastic Analysis for HALE Wings," 47th AIAA/ASME/ASCE/AHS/ASC Structures, Structural Dynamics, and Materials Conference, AIAA Paper 2006-1640, Newport, RI, USA, May 2006.

${ }^{24}$ Simo, J., "A finite strain beam formulation. The three-dimensional dynamic problem. Part I," Computer Methods in Applied Mechanics and Engineering, Vol. 49, No. 1, May 1985, pp. 55-70.

${ }^{25}$ Simo, J. C. and Vu-Quoc, L., "A three-dimensional finite-strain rod model. part II: Computational aspects," Computer Methods in Applied Mechanics and Engineering, Vol. 58, No. 1, Oct. 1986, pp. 79-116.

${ }^{26}$ Simo, J. C. and Vu-Quoc, L., "On the dynamics in space of rods undergoing large motions - A geometrically exact approach," Computer Methods in Applied Mechanics and Engineering, Vol. 66, No. 2, Feb. 1988, pp. 125-161.

${ }^{27}$ Cesnik, C. E. and Brown, E. L., "Modeling of high aspect ratio active flexible wings for roll control," Proceedings of the 43rd AIAA/ASME/ASCE/AHS/ASC Structures, Structural Dynamics and Materials Conference, AIAA, Denver, CO, 2002.

${ }^{28}$ Hodges, D. H., "Geometrically exact, intrinsic theory for dynamics of curved and twisted anisotropic beams," AIAA Journal, Vol. 41, No. 6, 2003, pp. 1131-1137.

${ }^{29}$ Sotoudeh, Z. and Hodges, D. H., "Incremental Method for Structural Analysis of Joined-Wing Aircraft," Journal of Aircraft, Vol. 48, No. 5, 2011, pp. 1588-1601.

${ }^{30}$ Palacios, R., "Nonlinear normal modes in an intrinsic theory of anisotropic beams," Journal of Sound and Vibration, Vol. 330, No. 8, April 2011, pp. 1772-1792.

${ }^{31}$ Murua, J., Palacios, R., and Graham, J. M. R., "Applications of the Unsteady Vortex-Lattice Method in Aircraft Aeroelasticity and Flight Dynamics," Progress in Aerospace Sciences, 2012, Commissioned.

${ }^{32}$ Hesse, H. and Palacios, R., "Consistent structural linearisation in flexible-body dynamics with large rigid-body motion," Computers 85 Structures, 2012, to appear.

${ }^{33}$ Murua, J., Hesse, H., Palacios, R., and Graham, J. M. R., "Stability and Open-Loop Dynamics of Very Flexible Aircraft Including Free-Wake Effects," 52 ${ }^{\text {nd }}$ AIAA/ASME/ASCE/AHS/ASC Structures, Structural Dynamics, and Materials Conference, AIAA 2011-1915, Denver, CO, USA, April 2011.

${ }^{34}$ Murua, J., Palacios, R., and Graham, J. M. R., "Open-Loop Stability and Closed-Loop Gust Alleviation on Flexible Aircraft Including Wake Modeling," 53 $3^{\text {rd }}$ AIAA/ASME/ASCE/AHS/ASC Structures, Structural Dynamics, and Materials Conference, Honolulu, HI, USA, April 2012.

${ }^{35}$ Géradin, M. and Cardona, A., Flexible multibody dynamics: a finite element approach, John Wiley and Sons, Inc., Chichester, UK, 2001.

${ }^{36}$ Hodges, D., "A mixed variational formulation based on exact intrinsic equations for dynamics of moving beams," International Journal of Solids and Structures, Vol. 26, No. 11, 1990, pp. 1253-1273.

${ }^{37}$ Stevens, B. L. and Lewis, F. L., Aircraft Control and Simulation, John Wiley \& Sons, Inc., New York, NY, USA, 1992.

${ }^{38}$ Hall, K., "Eigenanalysis of Unsteady Flows about Airfoils, Cascades and Wings," AIAA Journal, Vol. 32, No. 12, 1994, pp. 2426-2432.

${ }^{39}$ Waszak, M. R. and Schmidt, D. K., "Flight dynamics of aeroelastic vehicles," Journal of Aircraft, Vol. 25, No. 6, June 1988, pp. 563-571.

${ }^{40}$ Géradin, M. and Rixen, D., Mechanical Vibrations: Theory and Application to Structural Dynamics, Wiley-Blackwell, 2nd ed., May 1997. 